\title{
DEFORMAÇÕES EM SEDIMENTOS FINOS NÃO CONSOLIDADOS INTERPRETADAS COMO SISMITOS
}

JOÃO ARAÚJO-GOMES ${ }^{1}$

\begin{abstract}
Resumo - Este texto apresenta um resumo dos principais trabalhos internacionais sobre deformações em sedimentos finos não consolidados interpretadas como sismitos, e também uma proposta de esquematização ordenada de sismitos, com o objetivo de facilitar a sua leitura e interpretação. São ainda dadas noções básicas de deformação de sedimentos não consolidados, sismologia e liquefação de sedimentos.
\end{abstract}

Palavras-chave: Sismitos, SSD, liquefação.

\begin{abstract}
SOFT-SEDIMENT DEFORMATION INTERPRETED AS SEISMITES. This review is presented as a summary of the main international works on soft-sediment deformations (SSD) interpreted as seismites. It further includes a deformation schematic classification, based on the degree of seismic magnitude, in order to simplify the interpretation. Basic notions like unconsolidated sediment deformation, seismology, and sediment liquefaction are also taken into consideration.
\end{abstract}

Keywords: Seismites, SSD, liquefaction.

Résumé - DEFORMATION DE SEDIMENTS FINS NON CONSOLIDES, INTERPRETES COMME DES SEISMITES. Cette déformation a été l'objet de plusieurs articles internationaux dont la révision non exhaustive est présentée ici. Une classification des déformations selon le degré de magnitude séismique est proposée, afin de faciliter leur interprétation. Cette révision bibliographique présente aussi des concepts de base sur les déformations des sédiments non consolidés, ainsi que de séismologie et de liquéfaction des sédiments.

Mots clés: Seismites, SSD, liquéfaction.

Recebido: Novembro 2012. Aceite: Fevereiro 2013.

1 Bolseiro de Doutoramento FCT e Investigador do CEG-IGOT-UL, Núcleo SLIF - Sistemas Litorais e Fluviais: Dinâmicas, Mudanças Ambientais e Ordenamento do Território. E-mail: joaopgomes@campus.ul.pt 


\section{INTRODUÇÃO}

Esta síntese surge na sequência de um trabalho sobre Arqueossismologia efectuado no âmbito de um projeto da FCT (POCI/CTEGIN/58250/2004) (Gomes et al., 2008), que pretendeu referenciar, numa base de dados SIG, todos os sítios arqueológicos que pudessem ter sofrido deformação ou destruição sísmica. Este tema, então inédito em Portugal, apoiou-se nas evidências diretas e indiretas nos sítios arqueológicos, tendo-se recorrido à Paleossismologia como instrumento de análise principal.

Embora não fosse despropositado introduzir definições gerais de Arqueossismologia e Paleossismologia, até porque explicaria melhor o contexto deste trabalho, tal seria dispersante. Por isso começamos por um enquadramento do estudo das deformações de estruturas sedimentares secundárias, partindo depois para a proposta de listagem simplificada dessas deformações interpretadas como sismitos.

Antes de começar pelos exemplos de sismitos, há que fazer a distinção entre sismitos e deformações gerais de sedimentos: os sismitos ou sismoturbações são um tipo de deformação sedimentar. Existe uma série infindável de tipos de deformações, que não estão necessariamente associadas a uma origem sismogénica embora, desde Charles Lyell (1838), muitas destas deformações sedimentares tenham sido registadas e relacionadas com violentos episódios sísmicos. As deformações sedimentares são, no seu significado geral e segundo Mills (1983), deformações de uma unidade sedimentar não consolidada. A primeira está contida na segunda, e não o contrário.

Por outras palavras, um sismito é uma deformação litológica de origem sísmica. Só são aqui considerados sismitos as deformações em sedimentos finos não consolidados, mais conhecidos como soft-sediment deformations (Malkawi e Alawneh, 2000; Rodríguez-Pascua, 2000; Becker et al., 2002; Leroy et al., 2002; Bowman et al., 2004; Migowski et al., 2004; Weidlich e Bernecker, 2004; Schnellmann et al., 2005; Moretti e Sabato, 2007; Montenat et al., 2007; Deev et al., 2009; Suter et al., 2011, Moretti e Ronchi, 2011; Santos et al., 2012) que estejam diretamente relacionadas com a propagação de ondas sísmicas. Não consideramos as deformações em material mais grosseiro (Huang e Yu, 2013).

Embora sejam diversos os contextos geológicos onde um sismito se pode desenvolver, analisam-se aqui apenas os tipos de deformações sedimentares de origem sismogénica, que ocorrem geralmente em contextos sedimentares não litificados, pouco compactados e associados à presença de água (Montenat et al., 1993).

A deformação de sedimentos diz respeito a materiais não litificados (Mills, 1983). Se houver mecanismos impulsionadores (escoadas, tempestades e sismos) - reverse density gradient, slope failure, slumping or shear stress (Neuwerth, 2006: 81) -, suficientemente fortes para que o grau de compactação do sedimento seja reduzido, iniciando-se um processo de fluidização (idem), formar-se-ão testemunhos estruturais pós-deposicionais, que se podem reconhecer de uma forma relativamente fácil. A interpretação dessas estruturas será porventura mais difícil, pois as deformações sedimentares podem ser provocadas por diversos processos de perturbação, que não apenas os de origem sísmica (sismoturbações).

No entanto, e antes ainda de serem dados exemplos de sismitos, deve apresentar-se a definição dos que são já considerados genericamente como soft-sediment deformation (SSD - Suter et al., 2011), muitos deles interpretados como sismitos. Por Soft-sediment deformation (doravante, $\mathrm{SSD}$ ), entende-se uma assinatura sedimentar epigenética de deformação cossísmica. Mais concretamente é uma deformação pós-deposicional expressa por estruturas sedimentares secundárias, usualmente desenvolvidas em sedimentos de granulometria predominantemente fina, não consolidados e pouco compactados, e que, em grande parte dos casos, estão saturados de água. 
Normalmente quando são descritos SSD, estão-lhes associados fenómenos de fluidização e liquefacção - veremos mais à frente o que são - nos quais a água representa um papel fundamental. Uma longa lista de fenómenos de deformação sedimentar deste tipo (pós-deposicional, ou de deformação singenética e epigenética) vem sendo descrita em artigos de diversos investigadores, que se debruçam sobre o tema de SSD. Se nos quisermos focar, por exemplo, no tema da deformação de soft sediments interpretados como sismitos em ambientes lacustres, temos uma série de exemplos de trabalhos relacionados com SSD (Sims, 1973, 1975, 1976; Ben-Menahem, 1976; Hesse e Reading, 1978; Hempton e Dewey, 1983; Seilacher, 1984; Plint, 1985; El-Isa e Mustafa, 1986; Anand e Jain, 1987; Davenport e Ringrose, 1987; Scott e Price, 1988; Ringrose, 1989; Beck et al., 1992; Karlin e Abella, 1992; Van Loon et al., 1995; Beck et al., 1996; Alfaro et al., 1997; Lignier et al., 1998; Malkawi e Alawneh, 2000; Rodríguez Pascua et al., 2000; Becker et al., 2002; Leroy et al., 2002; Bowman et al., 2004; Migowski et al., 2004; Weidlich e Bernecker, 2004; Schnellmann et al., 2005; Mörner, 2005; Moreti e Sabato, 2007; Moretti e Ronchi, 2011).

Claro que há mais contextos, para além dos lacustres, onde este tipo de estudos se pode fazer. Mas não é por acaso que a maior parte deles dizem respeito a áreas costeiras, ou onde a presença de água é abundante porque, como veremos mais à frente, a liquefação e a fluidização dos sedimentos são das principais razões para que se forme este tipo de deformação. E isto acontece porque os sedimentos mais suscetíveis a deformação são os de granulometria fina (siltes e argilas), e em especial se tiverem disposição estratigráfica alternada de areias, siltes e argilas. É por esta razão que os SSD interpretados como sismitos se desenvolvem mais rapidamente em planícies aluviais, leitos de cheia, planícies deltaicas ou estuarinas, baías e ambientes lacustres ou palustres (Montenat et al., 2007: 9).

Grande parte dos cientistas que interpreta os SSD como sismitos, apesar de algumas opiniões contrárias, considera que só um sismo com magnitude igual ou superior a 4,5 na escala de Richter pode deixar registo de perturbação sedimentar. Se referirmos como exemplo as análises sísmicas efetuadas na região do vale de Cauca (formação de Zarzal na Colômbia), que indicam que os sismos que afetaram esta região terão atingido magnitudes compreendidas entre 5 e 7, verificamos que estão de acordo com as teorias de Scott e Price, que sugerem que uma magnitude sísmica inferior a 5 não é suficientemente forte para causar liquefação para além da distância de $4 \mathrm{~km}$ do epicentro (Scott e Price, 1988). No entanto, estes autores também afirmam, que uma magnitude de 7 na escala de Richter não significa obrigatoriamente que os sedimentos situados a $20 \mathrm{~km}$ de distância da fonte sísmica venham a apresentar sinais de sismoturbação (Neuwerth et al., 2006).

Relacionando estes dados, por exemplo, com as possíveis falhas ativas afetando a formação de Zarzal, observa-se uma grande concordância de resultados que podem ser transpostos para análises e extrapolações de situações similares, em que as deformações de estruturas sedimentares estejam próximas - menos de $20 \mathrm{~km}$ - de falhas ativas (Neuwerth, 2006). A relação do sismito com a distância à falha sismogénica e a magnitude do sismo é sempre tida em conta em estudos sobre sismitos e SSD (Galli, 2000; Obermeier et al., 2005).

\section{PRINCIPAIS TIPOS DE SSD INTERPRETADAS COMO SISMITOS}

Não se pretende aqui uma análise detalhada dos mecanismos impulsionadores para se efetuar uma classificação detalhada dos sismitos - embora trabalhos mais recentes venham reforçar essa necessidade (Ghosh et al., 2012). Pretende-se, isso sim, a explanação tipológica dessas deformações de maneira a ficarmos com a noção do que deve ser considerado como sismito, e do que deve ser interpretado apenas como SSD de origem não sísmica. 
As definições mais frequentes, a seguir apresentadas, dos tipos de deformação sedimentar pós-deposicional interpretadas como sismitos são uma lista de sismoturbações que normalmente ocorrem em sedimentos não consolidados e litificados, e que, de maneira nenhuma, pretendem representar o panorama global de todos os tipos de SSD. Esta classificação apresenta-se como um sistema aberto, dentro do qual serão certamente acrescentados novos exemplos de assinaturas sedimentares deste género, à medida que crescer o número de trabalhos sobre formações sedimentares não consolidadas.

Para além disso, os exemplos seguintes apenas serão referentes a tipologias de deformações de sedimentos de idade relativamente recente. Estão pois excluídas desta lista de SSD, não só os sismitos que se reportem a períodos anteriores aos estudados pela Paleossismologia e Neotectónica, mas também àqueles que não se insiram num contexto de proximidade costeira.

Organizados a partir duma lógica crescente de grau de liquefação - veremos mais à frente do que se trata - apresentaremos primeiro exemplos de sismitos sem sinais de liquefação (normalmente associados a casos de microssismicidade ou de sismos de magnitude inferior ou igual a 5 na escala de Richter), e depois os que apresentem evidências de liquefação (casos de associação a sismos de magnitude elevada, a partir da magnitude 6 na escala de Richter).

\section{Estruturas sedimentares de deformação cossísmica sem liquefação de sedimentos}

Por estruturas sedimentares de deformação entendem-se todas as modificações estruturais das estratificações sedimentares que apresentem sinais de terem sido induzidas por um agente externo suficientemente perturbador. Isto é, são estruturas que não apresentam características de sedimentação normal e indiciam episódios de perturbação mecânica.

\subsection{Loop Bending (laços)}

Pode entender-se o fenómeno de loop bending como uma curvatura das sucessivas laminações estratigráficas de sedimentos não litificados, resultantes da distensão dos sedimentos, induzida por pequenos e repetidos tremores de terra (Rodríguez-Pascua et al., 2000). Por outro lado, os diferentes tipos de "laços", para além de indicarem o tipo de deformação que as laminações adotaram durante os tremores de terra, podem informar, por vezes, sobre a direção das vibrações sísmicas (Rodríguez-Pascua et al., 2000). Este tipo de deformação é de origem cossísmica direta, o que é o mesmo que dizer que foram as ondas sísmicas e movimentos distensivos resultantes da sua ação que originaram a deformação (fig. 1a; fig. 2a).

\subsection{Perturbações laminares}

São pequenas perturbações onduladas, em sedimentos finos laminados anteriormente plano-paralelos, que podem atingir $10 \mathrm{~cm}$ de espessura. Este tipo de SSD pode ser interpretado como sismito, mas pode ter outra origem para além da sísmica (por exemplo, a ação gravítica de uma unidade suprajacente pode também estar na origem destas estruturas sedimentares) (fig. 1b; fig. 2b).

\subsection{Estratificações convolutas sem liquefação de sedimentos}

Estas estruturas não são muito frequentes, mas podem ser visíveis em areias de grão fino e médio, e a sua espessura pode chegar aos $20 \mathrm{~cm}$. As dobras, muito contorcidas, formam 
pequenos sinformas e antiformas, e estão associadas a estruturas de carga, slumps, e intrusões de sedimentos (Neuwerth et al., 2006, fig. 8: 79). Como no exemplo anterior, também estas SSD podem ter outras origens para além das sísmicas (fig. 1c; fig. 2c).

\subsection{Slumpings sem liquefação de sedimentos.}

Visíveis normalmente em sedimentos finos (argilas, siltes, areias de grão fino e médio) mais suscetíveis a perturbações gravíticas, estas deformações podem atingir mais de um metro de amplitude. O plano axial das dobras causadas pelo slumping (laminação convoluta) pode ter inclinações variadas (Neuwerth et al., 2006, fig. 8, p 80). Estão frequentemente associados a estruturas de carga (load structures). Se analisados individualmente não constituem evidências fidedignas de sismicidade (fig. 1d; fig. 2d).

\section{Estruturas sedimentares de deformação com liquefação de sedimentos}

The main coseismic geological effects which may damage buildings are liquefaction, lateral spreading and landsliding. Liquefaction, with or without lateral spreading, may occur many kilometres away from an epicentral area (Galli, 2000), [e] may cause foundation displacement and buildings to collapse. (Galadini et al., 2006: 400).

A liquefação corresponde à passagem de um sedimento do estado sólido ao estado fluido, com consequente perda de capacidade de suporte, em resultado do aumento, dinamicamente induzido, da pressão do fluido intersticial (Galadini et al., 2006: 400).

A partir do momento em que um determinado local é violentamente perturbado, quer por um forte abalo sísmico, quer por qualquer outro evento violento de agitação terrestre, pode haver liquefação ou fluidificação dos sedimentos. Se essa liquefação ocorrer poderá desencadear uma fluidização ou liquidização sedimentar, o que é o mesmo que dizer a circulação de um determinado elemento sólido através de um agente líquido de transporte ou seja, arraste das partículas sedimentares por fluido sob pressão (tipicamente água), que circula no sedimento em direção a zonas de menor pressão.

Há que explicar o sentido atribuído aos termos fluidização e fluidificação, bem como liquefação e "liquidização". Entendemos que liquefação e fluidificação são a mesma coisa, assim como fluidização e "liquidização". No entanto, liquefação ou fluidificação não são o mesmo que liquidização ou fluidização. A primeira origina a segunda.

Atendamos, por isso, ao facto de as próximas definições pressuporem sempre a existência de fluidização sedimentar, uma vez que nos estamos a referir a sedimentos anteriormente liquefeitos, ou fluidificados.

\subsection{Figuras de carga (Load Structures)}

As load structures ou load cast structures, estruturas (ou figuras) de carga, são normalmente estruturas arredondadas, que se desenvolvem na vertical, correspondendo a penetrações de sedimentos superiores, mais densos, em sedimentos inferiores e menos densos (Rodríguez-Pascua, 2000; Becker et al., 2002; Leroy et al., 2002; Bowman et al., 2004; Migowski et al., 2004; Weidlich e Bernecker, 2004; Schnellmann et al., 2005; Moretti e Sabato, 2007; Montenat et al., 2007; Deev et al., 2009; Suter et al., 2011, Moretti e Ronchi, 2011). Representam pequenas subsidências ou abatimentos nos sedimentos que, no caso de 
um evento súbito de deformação pós-deposicional, prolongam verticalmente pequenas ondulações pré-existentes. Podem estar associadas a duas origens: gravítica simples, ou desencadeada por vibrações sísmicas (fig. 1e; fig. 2e).

\subsubsection{Estruturas em pêndulo (Pendulous form Structures)}

Frequentemente encontradas em sedimentos arenosos, assentes sobre argilas e materiais mais finos e, por isso, menos densos, este tipo de estrutura tem a base sub-planar e assemelha-se a um lóbulo convexo apontado para baixo (Neuwerth et al., 2006: 74). São uma variante do exemplo anterior e têm a mesma origem. Também esta deformação sedimentar pode ter a ação gravítica como mecanismo impulsionador exclusivo, para além do sísmico (fig. 1f).

\subsubsection{Estruturas em chama (Flame Structure)}

O tamanho varia entre os centímetros e os decímetros e, na maior parte dos casos, são muito pouco nítidas num corte estratigráfico. Manifestam-se normalmente em siltes e materiais mais finos como a argila. Os sismos e a gravidade promovida pelo peso das unidades superiores podem estar na origem desta deformação (fig. 1g).

\subsection{Pseudo nódulos e nódulos (Pseudonodules - attached and detached)}

Embora sejam mais raras, estas deformações sedimentares mostram, no seu interior, material do sedimento suprajacente. Os pseudo nódulos correspondem a estruturas de carga em vias de se destacarem, ou já destacadas, das unidades sedimentares de origem, por avanço pronunciado da subsidência no interior do nível sedimentar subjacente, menos denso (Neuwerth et al., 2006, fig. 8: 76; Suter et al., 2011: fig. 7: 196). Revelam maior fluidez de sedimentos, ou seja menor viscosidade, indiciando maior probabilidade de origem sísmica.

Tanto uns como outros se manifestam em areias de grão médio e fino intercaladas por argila, ou em areias finas quando, por exemplo, os pseudo nódulos estão associados a movimentos arenosos ascendentes ou de laminação convoluta associados a fluidização (Neuwerth et al., 2006, fig. 8: 76-77) (fig. 1h e i; fig. 2h e i).

Trabalhos recentes efetuados na zona de Belém, Lisboa, mostram que também se podem formar nódulos perfeitos através do processo de liquefação (Ramos-Pereira et al., neste volume).

Estes SSD são excelentes indicadores sísmicos porque estão, como referimos, associados outros tipos de sismitos. Isoladamente, contudo, são pouco claros quanto à sua génese (fig. 1g; fig. 2j).

\subsection{Estruturas de escape de água (Water Escape Structures)}

O escape de água por ação sísmica em sedimentos, conduzindo ao arraste de partículas e associado à fluidização, caracteriza-se por subidas repentinas de água intersticial existente nesses sedimentos. Ao haver movimentos cíclicos do material sedimentar, desencadeados pela passagem das ondas sísmicas, a água existente na porosidade sofre um aumento de pressão por densificação do material granular (desencadeando liquefação) e tende a ascender rapidamente, deformando a componente sedimentar onde está inserida e a imediatamente 
superior. Assim, se um sedimento for suscetível à liquefação, a água intersticial pode ser expelida, deixando marcas desse fluxo repentino de ejeção. As marcas de ejeção podem ser verticais ou oblíquas, isto é, transversais em relação à estratificação, ou horizontais ou seja, paralelas à estratificação (Ricci Lucchi, 1992: 147-148). Por vezes podem ser confundidas com as estruturas de carga em forma de chama - flame structure. As estruturas de escape de água podem apresentar também deformação interna, uma vez que são provocadas pelo movimento ascendente da água. No entanto, estas não são deformadas a partir de uma posição superior pelo enchimento ou abatimento dos sedimentos sobrepostos.

\subsubsection{Estrutura cúspida (Water escape cusp)}

$\mathrm{Na}$ sua morfologia e aparência são semelhantes às estruturas de carga em flame structure. Porém, como contêm sedimentos inferiores deformados pela expulsão de água, são distintas e de formação diferente. (Neuwerth et al., 2006: 78) (fig. 2k).

\subsubsection{Variações morfológicas e Pillar Structures}

As pillar structures são definidas e classificadas pela sua morfologia. Isto é, depois de ter havido ejeção de água, a orientação estratigráfica dos sedimentos fica diretamente relacionada com o sentido do movimento da água ejetada - movimento ascendente, dando à estrutura sedimentar um aspeto de coluna (pillar).

Para além disso, este tipo de estrutura sedimentar pode originar outros tipos de deformações. As mais frequentes são as associações de dish-and-pillar structure (prato-coluna) e pocket-and-pillar structure (bolsa-coluna). Estes dois exemplos estão por sua vez frequentemente associados às laminações de estratificação convolutas (convolute laminations) e às intrusões de sedimentos (fig. 21 e m).

Estas deformações sedimentares estão associadas a eventos sísmicos cujo foco de origem pode ser bem determinado. Consideramos por isso que são boas indicadoras de deformação cossímica.

\section{Estruturas de intrusão de sedimentos}

\subsection{Injeções de areia}

As injeções de areia são indicadoras de um episódio de deformação intensa do sedimento (observáveis em corte estratigráfico). Podendo assumir diversas formas (diques verticais ou com pequenas derivações perpendiculares entrecruzadas e atingir alguns metros de extensão ou e soleiras horizontais), estas estruturas estão associadas a processos caracterizados por movimentações ascendentes ou descendentes de areias mais grosseiras em sedimentos mais finos. Quando correspondem a movimento de injeção ascendente, constituem-se como estruturas pós-deposicionais indicadoras de fortes vibrações sísmicas.

Note-se que a presença de filões de areia em sedimentos mais finos subjacentes pode ter origem em processos gravíticos, uma vez que os sedimentos arenosos podem preencher fendas nos sedimentos (Galopim de Carvalho, 2005: 90). Segundo Galopim de Carvalho, os fenómenos sísmicos podem não ser os principais responsáveis por essa penetração de areia mais recente em sedimentos finos mais antigos. Julgamos, no entanto, que a movimentação ascendente da areia, mais antiga, para sedimentos mais recentes, só pode ser fruto de um 
violento abalo terrestre. Por tudo isto, devemos entender o termo injeção como intrusão ascendente de areia, seja ela fina ou grosseira, havendo casos em que essa intrusão tem origem sísmica. (fig. 1f; fig. 2o).

\subsection{Injeção de material mais grosseiro (areia e cascalho)}

Neste tipo de intrusão sedimentar, o fenómeno observado é em tudo semelhante ao da injeção de areias. A grande diferença é que a sua composição sedimentar é variável, consisting of predominantly coarse-grained and lesser medium-grained sands (Neuwerth et al., 2006, fig. 8: 78) (fig. 2p).

\subsection{Estruturas em almofada (Pillow Structures)}

Este tipo de estruturas é representado por deformações côncavas em sentido descendente, desencadeadas normalmente pela fluidização e ascenção de sedimentos arenosos inferiores (Rodríguez-Pascua et al., 2000: 125-126) (fig. 1i; fig. 2n).

\section{OUTROS TIPOS DE SSD}

Existem outros tipos de deformações sedimentares provocados pela fluidização, com outras variáveis morfológicas. São fenómenos muito semelhantes de fluidização ascendente, mas que se manifestam em sedimentos de granulometria mais fina - silte - e em escala bastante mais reduzida. É, por exemplo, o caso de pequenas intrusões ascendentes de material fino em sedimentos plano-paralelos laminados, que podem assumir formas bastantes peculiares (Deev et al., 2009), como os mushroom-like silts (Rodríguez-Pascua et al., 2000) ou as rolled-up structures (Suter et al., 2011).

É necessário ter em conta, portanto, que o mecanismo sísmico desencadeador destas deformações de sedimentos não consolidados pode fazer variar o padrão de deformação segundo a intensidade das vibrações no local. Contudo, o tipo de deformação sedimentar não depende exclusivamente da intensidade sísmica que a gera: depende também do próprio contexto sedimentar em que se insere, nomeadamente o potencial de liquefação dos sedimentos presentes.

Há que atender a que as deformações sedimentares relacionadas com fracas intensidades sísmicas, anteriormente referidas, nomeadamente as perturbações laminares e as laminações dobradas sem liquefação, podem ser também geradas por outros mecanismos (como por exemplo uma unidade estratigráfica mais densa suprajacente). Por isso mesmo é precipitado classificar como sismito uma deformação sedimentar deste género se for encontrada isolada numa determinada camada estratigráfica sem mais deformações coevas associadas.

Para facilitar a compreensão global do tipo de deformação sedimentar relacionada com a intensidade sísmica que lhe dá origem, por sua vez função da magnitude do sismo e da distância à falha geradora, propõe-se um esquema sinóptico de sismoturbações sedimentares (fig. 1), onde simulámos o que aconteceria se sedimentos iguais fossem deformados por sismos de magnitudes diferentes (e consequentemente por diferentes intensidades). Alertamos para o facto de a escala vertical do esquema não representar profundidade, mas a relação entre a severidade das vibrações sísmicas (intensidade) e a sismoturbação desencadeada. Para a construção deste esquema considerou-se também a mesma distância epicêntrica teórica, pois sabe-se que a sismoturbação não está apenas associada com a magnitude e a intensidade sísmica, mas também com a distância ao epicentro sísmico. 

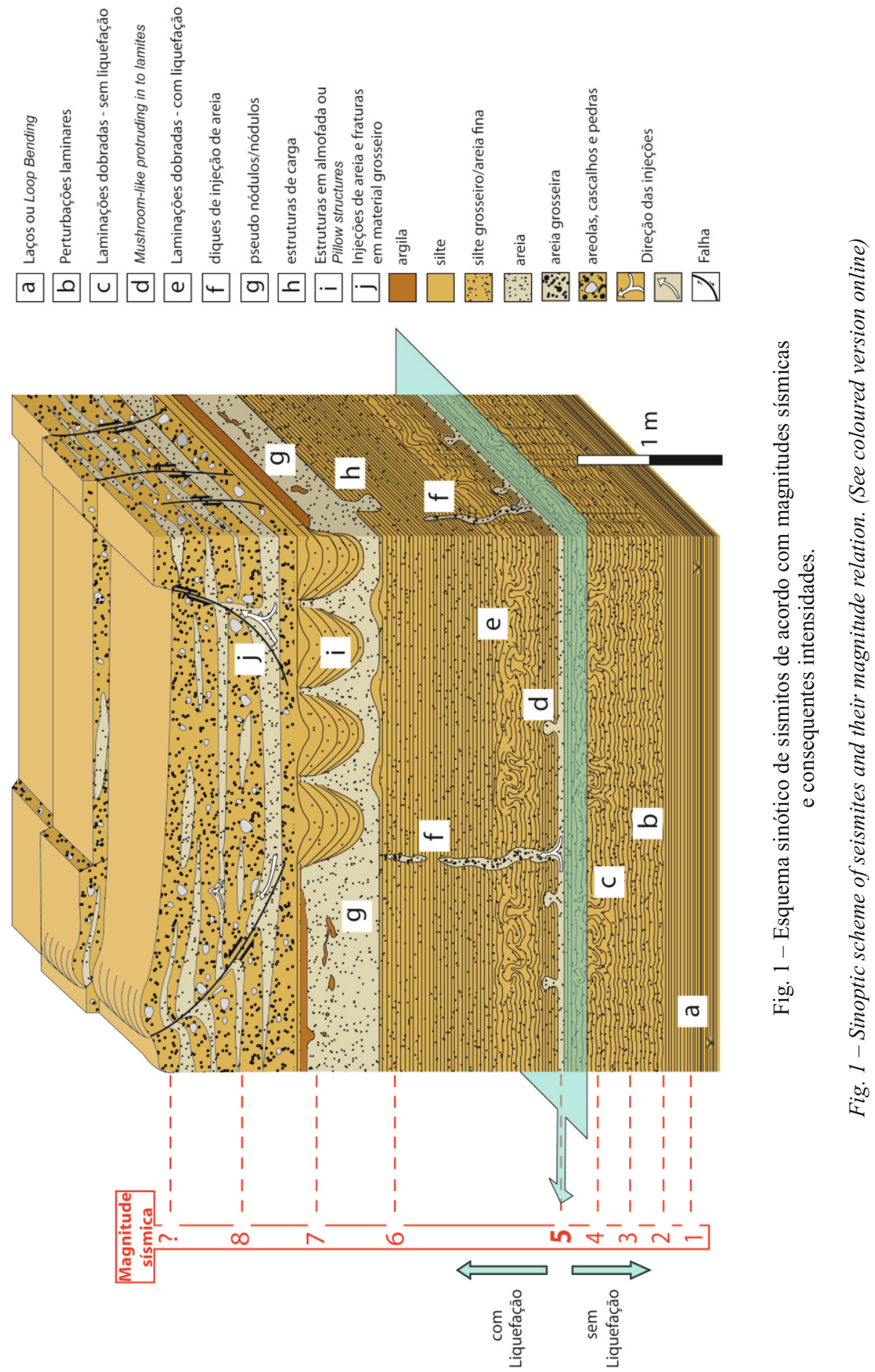


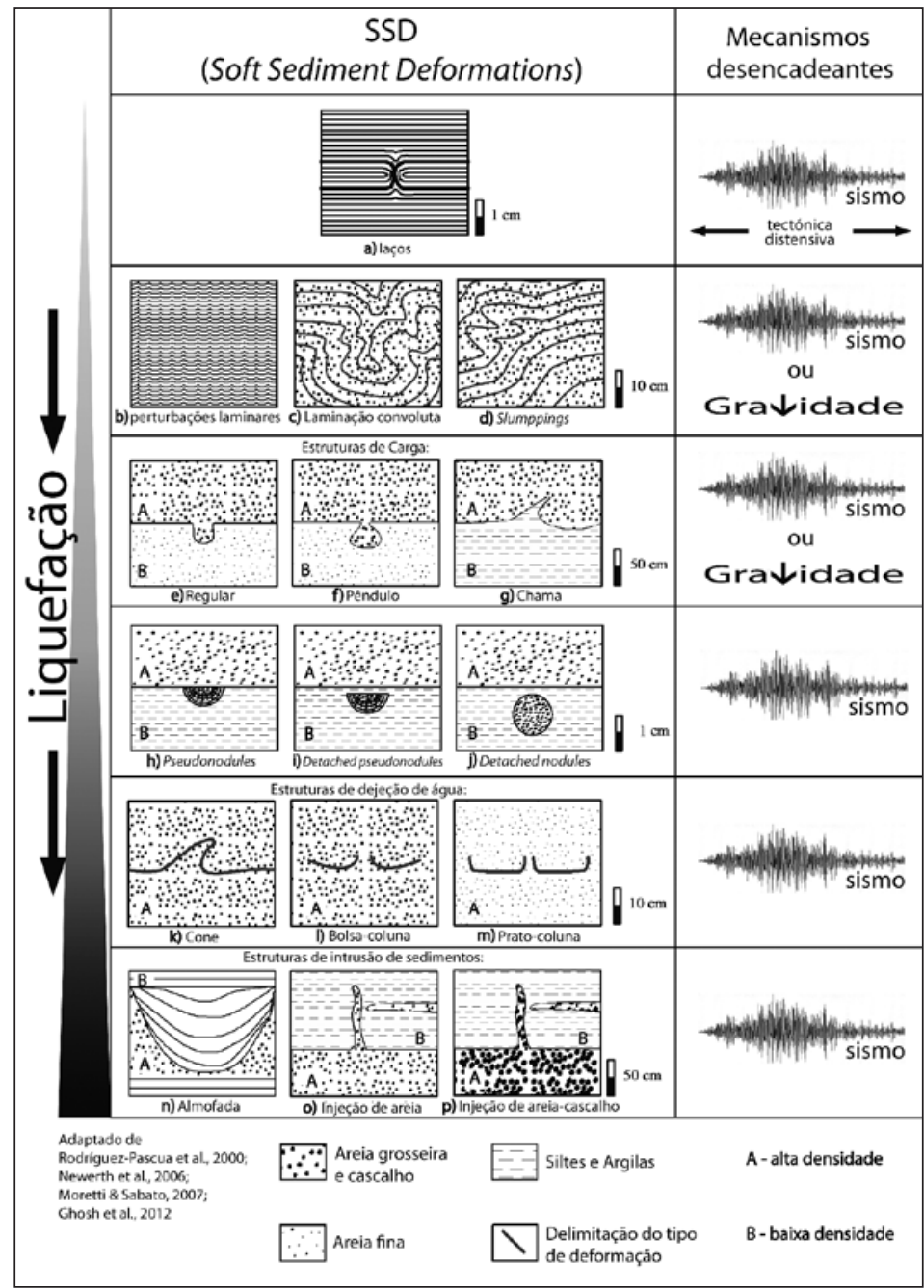

Fig. 2 - Esquema interpretativo da relação entre SSD, grau de deformação e mecanismos desencadeantes.

Fig. 2 -Relation Scheme between Soft-sediment deformation, liquefaction e triggering mechanisms.

\section{CONCLUSÃO}

Nesta análise da deformação em sedimentos finos não consolidados interpretada como sismitos, houve a consciência de existirem muitos mais exemplos de deformações sedimentares aqui não referidos, ou não fosse esta uma tentativa de reunir opiniões dos principais trabalhos realizados para o tema. De facto, há uma grande diversidade de parâmetros contextuais que fornecem às deformações deste género uma quase impossibilidade de repetição dimensional e tipológica. O que aqui se abordou foram os principais padrões de SSD e a 
maneira como eles são interpretados. Eles podem constituir-se como excelentes indicadores de paleossismos - podendo informar não só da magnitude, mas também da intensidade das vibrações sísmicas ocorridas e, consequentemente, colocar hipóteses plausíveis à distância à fonte sísmica (falha ativa geradora) e à magnitude do sismo.

Esta proposta de lista simplificada apenas aborda os artigos considerados essenciais, para uma abordagem sedimentar inicial e não esconde o papel pouco preponderante da novidade científica num plano internacional. Sabe-se, no entanto e apesar da existência de alguns trabalhos recentes sobre evidências em deformação de sedimentos (Dinis et al. 2007, Ressurreição et al., 2009, Ressurreição et al., 2011), que no plano nacional pouco tem sido avançado relativamente ao estudo das deformações de estruturas sedimentares não consolidadas de origem sísmica.

Por isso mesmo, esta recensão pretende contribuir para a difusão bibliográfica relacionada com a temática dos sismitos, fornecendo uma lista de mecanismos de identificação sedimentar, a partir de casos estudados e reforça a importância da multidisciplinaridade neste tipo de estudos.

Referiu-se a característica comum nos sismitos, que se prende com o papel decisivo da água para a sua génese. Para além deste fator, viu-se que é necessária uma magnitude igual ou superior 5 para haver liquefação de sedimentos, assim como os sismitos estarem normalmente associados a falhas ativas próximas, para que a intensidade das vibrações sísmicas seja suficientemente forte. Atribui-se, pois, aos sismitos um papel de extrema importância nos estudos da Paleossismologia e Neotectónica, bem como em Geomorfologia, permitindo identificar e datar eventos sísmicos não registados (paleossismos).

Seja num contexto lacustre, de planície aluvial, de zona estuarina ou até mesmo de praia, a deformação epigenética de sedimentos, com origem sísmica, não será, em princípio, uma manifestação isolada, nem terá apenas uma forma. Geralmente vários tipos de SSD encontram-se associados entre si, embora circunscritos a um conjunto sedimentar coevo e localizado. Por isso mesmo, devemos entender esta catalogação sobretudo como instrumento indicador de sistema aberto e não como ferramenta que permite incluir a análise de sismoturbações.

Embora seja feita uma tentativa de individualização dos vários tipos de sismitos, os SSD só podem devem ser interpretados num contexto sedimentar global e nunca individualizados na sua tipologia de análise (Ghosh et al., 2012). Isto é, depois de analisados em pormenor, devem ser inseridos num estudo contextual e preferencialmente multidisciplinar.

Um sismo com magnitude suficientemente forte para perturbar estruturas sedimentares é provavelmente capaz de assinar (deformações) de várias maneiras, dependendo do suporte (sedimentar) onde escreve.

\section{AGRADECIMENTOS}

À Professora Doutora Ana Ramos Pereira do Centro de Estudos Geográficos, que contribuiu para a prossecução deste tema, em que pensava desde o início da minha formação profissional, e me permitiu efetivar a interdisciplinaridade. Quero também agradecer ao Laboratório de Tectonofísica e Tectónica Experimental da Faculdade de Ciências da Universidade de Lisboa por ter patrocinado o primeiro projeto de Arqueossismologia do país.

Agradeço finalmente a revisão científica deste trabalho, que melhorou bastante o seu conteúdo e a sua leitura. 


\section{BIBLIOGRAFIA}

Alfaro P, Moretti M, Soria, J M (1997) Soft-sediment deformation structures induced by earthquakes (seismites) in Pliocene lacustrine deposits (Guadix-Baza Basin, Central Betic Cordillera). Eclogae Geol. Helv, 90: 531-540 .

Anand A, Jain A K (1987) Earthquakes and deformational structures (seismites) in Holocene sediments from the Himalayan-Andaman Arc, India. Tectonophysics, 133: 105-120.

Beck C, Frédéric M, Chapron E, Van Rensbergen P, De Batist M (1996) Enhanced seismicity in the early post-glacial period: evidence from the Post-Würm sediments of Lake Annency, north-western Alps. Journ. Geodyn., 22 (1/2): 155-171.

Becker A, Colin A, Davenport C A, Giardini D (2002) Palaeoseismicity studies on end-Pleistocene and Holocene lake deposits around Basle, Switzerland. Geophys. Journ. Int., 149: 659-678.

Ben-Menahem A (1976) Dating of historical earthquakes bymud profiles of lake-bottom sediments. Nature, 262: 200-202.

Bowman D, Korjenkov A, Porat N (2004) Late-Pleistocene seismites from Lake Issyk-Kul, the Tien Shan range, Kyrghyzstan. Sediment. Geol. 163, 211-228.

Cabral J (1993) Neotectónica de Portugal Continental. Dissertação de Doutoramente em Geologia, na especialidade de Geodinâmica Interna, Dep. Geologia, Fac. Ciências da Univ. de Lisboa.

Davenport C A, Ringrose P S (1987) Deformation of Scottish Quaternary sediment sequences by strong earthquake motion. In Jones $\mathrm{M} \mathrm{E}$, Preston R M F (Eds.) Deformation of sediments and sedimentary rocks. Geological Society Spec. Publ., 29: 299-314.

Deev E V, Zolnikov I D, Gus'kov S A (2009) Seismites in Quaternary sediments of southeastern Altai. Russian Geology and Geophysics, 50: 546-561.

Dinis P, Cabral J, Soares A F (2007) Genesis of deformation structures affecting Plio-Pleistocene sediments in the western Portuguese mainland (West Iberia). Implication on the regional neotectonics. Geodinamica Acta 20/6: 415-431.
El-Isa Z H, Mustafa H (1986) Earthquake deformations in the Lisan deposits and seismotectonic implications. Geophys. J. R. Astron. Soc. 86, 413-424.

Galadini F, Hinzen K G, Stiros S (2006) Archeoseismology: methodological issues and procedure. Journal of Seismology, 10: 395-414.

Galli P (2000) New empirical relationships between magnitude and distance for liquefaction. Tectonophysics, 324: 169-187.

Galopim de Carvalho A M (2005) Geologia sedimentar. Sedimentogénese. Âncora Ed., vol. II.

Ghosh S K, Pandey A K, Pandey P, Ray Y, Sinha S (2012) Soft-sediment deformation structures from the Paleoproterozoic Damtha Group of Garhwal Lesser Himalaya, India. Sediment. Geol., 261-262: 76-89.

Gomes J A, Angelucci D E, Cabral J (2008) Arqueossismologia: estado actual do conhecimento em Portugal. Comunicações Geológicas, 95: 73-92.

Hempton M R, Dewey J F (1983) Earthquake-induced deformational structures in young lacustrine sediments, East Anatolian Fault, southeast Turkey. Tectonophysics, 98: 7-14.

Hesse R, Reading H G (1978) Subaqueous clastic fissures and other examples of sedimentary transposition in the lacustrine Horton Bluff Formation (Mississippian), Nova Scotia, Canada. Spec. Publ. Int. Ass. Sediment. 2: 241-257.

Huang Y, Yu M (2013) Review of soil liquefaction characteristics during major earthquakes of the twenty-first century. Natural Hazards, 65: 2375-2384.

Karlin R E, Abella S E B (1992) Paleoearthquakes in the Pugeot Sound Region recorded in sediments from lakeWashington, U.S.A. Science 258: 1617-1619.

Keller E A, Pinter N (2002) Active tectonics. Prentice Hall, Upper Saddle Rivers, 2nd ed.

Leroy S, Kazanc N, Ileri O, Kibar M, Emre O, McGee E, Griffiths H I (2002) Abrupt environmental changes within a late Holocene lacustrine sequence south of the Marmara Sea (Lake Manyas, N-W Turkey): possible links with seismic events. Mar. Geol. 190: 531-552. 
Lignier V, Beck C, Chapron E (1998) Caractérisation géométrique et texturale de perturbations synsédimentaires attribuées à des séismes, dans une formation quaternaire glaciolacustre des Alpes (les Argiles du Trièves). C. R. Acad. Sci., 327: 645-652.

Lyell C (1838) Elements of geology. Open Library. org: http://www.archive.org/stream/elementsgeochar00lyelrich.

Malkawi A I H, Alawneh A S (2000) Paleoearthquake features as indicators of potential earthquake activities in the Karameh Dam Site. Nat. Hazards, 22: 1-16.

Migowski C, Agnon A, Bookman R, Negendank J F W, Stein M (2004) Recurrence pattern of Holocene earthquakes along the Dead Sea transform revealed by varve-counting and radiocarbon dating of lacustrine sediments, Earth Planet. Sci. Lett., 222: 301-314.

Mills P C (1983) Genesis and diagnostic value of soft-sediment deformation structures (seismites) around Sumdo in the lower Spiti valley (thethys Himalya). Sediment. Geol., 101: 69-83.

Montenat C, Barrier P, Ott d'Estevou P, Hibsch C (2007) Seimites: an attempt at critical analysis and classification. Sediment. Geol., 196: 5-30.

Montenat C, Ott d'Estevou P, Barrier P, Paturel D (1993) Les séismites: essai de typologie génétique. Géochronique, 46, 15-17.

Moretti M (2000) Soft-sediment deformation structures interpreted as seismites in middle-late Pleistocene aeolian deposits (Apulian foreland, southern Italy). Sediment. Geol., 135: 167-179.

Moretti M, Sabato L (2007) Recognition of trigger mechanisms for soft-sediment deformation in the Pleistocene lacustrine deposits of the Sant Arcangelo Basin (Southern Italy): seismic shock vs. overloading. Sediment. Geol., 196: 31-45.

Moretti M, Ronchi A (2011) Liquefaction features interpreted as seismites in the Pleistocene fluvio-lacustrine deposits of the Neuquén Basin (Northern Patagonia). Sediment. Geol., 235: 200-209.

Neuwerth R, Suter F, Guzman C A, Gorin G E (2006) Soft-sediment deformation in a tectonically active area: the Plio-Pleistocene Zarzal Formation in the Cauca Valley (Western Colombia). Sediment. Geol., 186: 67-88.
Obermeier S F (1994) Using liquefaction-induced features for paleoseismic analysis. Ch. A. In Obermeier S F, Jibson R W (Eds.) Using groundfailure features for paleoseismic analysis. Geological Survey Open-File Report: 94-633: A1-A98.

Obermeier S F, Olson S M, Green R A (2005) Field occurrences of liquefaction-induced features: a primer for engineering geologic analysis of paleoseismic shaking. Engineering Geol., 76: 209-234

Owen G (1987) Deformation processes in unconsolidated sands. In Jones M E, Preston R M F (Eds.) Deformation of sediments and sedimentary rocks. Geol. Soc. Spec. Publ., 29: 11-24.

Plint A G (1985) Possible earthquake-induced soft-sediment faulting and remobilization in Pennsylvanian alluvial strata, southern New Brunswick. Canada, Can. J. Earth Sci., 22: 907-912.

Ramos-Pereira A, Trindade J, Araújo-Gomes J (2013) Seismite and Tsunamiite in Urban Environment (Lisbon). Finisterra - Revista Portuguesa de Geografia. XLVIII (95): 141-147.

Ressurreição R, Cabral J, Dias R (2009) Estudos de neotectónica na Falha de Carcavai (Algarve): evidências de deformação em sedimentos plio-quaternários. In Boski T, Moura D, Gomes A (Eds) Livro de Resumos, VII Reunião do Quaternário Ibérico, Faro, Portugal, 5 a 9 de Outubro de 2009, CIMA, Universidade do Algarve: 59-62.

Ressurreição R, Cabral J, Dias R P, Carvalho J, Pinto C (2011) Neotectonic activity on the Carcavai fault zone (Algarve, Portugal). Comunicações Geológicas, 98: 5-14.

Ricci Lucchi F (1992) Sedimentografia. Atlante fotografico delle strutture sedimentarie. Zanichelli, Bologna, $2^{a} \mathrm{Ed}$.

Ringrose P S (1989) Paleoseismic (?) liquefaction event in late Quaternary lake sediment at Glen Roy, Scotland. Terra Nova, 1: 57-62.

Rodríguez-Pascua M A, Calvo J P, de Vicente G, Gómez-Gras D (2000) Soft-sediment deformation structures interpreted as seismites in lacustrine sediments of the Prebetic Zone, SE Spain, and their potential use as indicatores of earthquake magnitudes during the Late Miocene. Sediment. Geol. 135: 117-135.

Santos M, Almeida R P, Mountney N P, Fragoso-Cesar A (2012) Seismites as a tool in the pa- 
laeoenvironmental reconstruction of fluvial deposits: the Cambrian Guarda Velha Formation, southern Brazil. Sediment. Geol., 277-278: 52-60.

Schnellmann M, Anselmetti F, Giardini D, McKenzie J (2005) Mass movement-induced fold-and-thrust belt structures in unconsolidated sediments in Lake Lucerne (Switzerland). Sedimentology 52(2): 271-289.

Scott B, Price S (1988) Earthquake-induce structures in young sediments. Tectonophysics, 147: 165-170.

Seilacher A (1984) Sedimentary structures tentatively attributed to seismic events, Mar. Geol., 55: 1-12.

Seilacher A (1969) Fault-graded beds interpreted as seismites, Sedimentology, 13: 155-159.

Sims J D (1976) Environments of earthquake-induced deformational structures in sediments. Trans. Am. Geophys. Union 63, 435 Abstract.
Sims J D (1975) Determining earthquake recurrence intervals from deformational structures in young lacustrine sediments. Tectonophysics, 29: 141-152.

Sims J D (1973) Earthquake-induced structures in sediments of Van Norman Lake, San Fernando, California. Science, 182: 161-163.

Suter F, Martínez J I, Vélez M I (2011) Holocene soft-sediment deformation of the Santa $\mathrm{Fe}-$ Sopetrán Basin, northern Colombian Andes: Evidence for pre-Hispanic seismic activity? Sediment. Geol. 235: 188-199.

Van Loon A J, Brodzikowski K, Zielinski T (1995) Shock-induced resuspension deposits from a Pleistocene proglacial lake (Kleszców Graben, central Poland). Journ. Sediment. Res., A65(2): 417-422.

Weidlich O, Bernecker M (2004) Quantification of depositional changes and paleo-seismic activities from laminated sediments using outcrop data. Sediment. Geol., 166: 11-20.

O autor deste texto escreve segundo o novo acordo ortográfico 